J16021+3326: NEW MULTI-FREQUENCY OBSERVATIONS OF A COMPLEX SOURCE

This article has been downloaded from IOPscience. Please scroll down to see the full text article.

2010 ApJ 712159

(http://iopscience.iop.org/0004-637X/712/1/159)

The Table of Contents and more related content is available

Download details:

IP Address: 131.215.193.213

The article was downloaded on 26/03/2010 at 21:27

Please note that terms and conditions apply. 


\title{
J16021+3326: NEW MULTI-FREQUENCY OBSERVATIONS OF A COMPLEX SOURCE
}

\author{
S. E. Tremblay ${ }^{1}$, G. B. Taylor ${ }^{1,2}$, J. L. Richards ${ }^{3}$, A. C. S. Readhead ${ }^{3}$, J. F. Helmboldt ${ }^{4}$, R. W. Romani ${ }^{5}$, And S. E. Healey ${ }^{5}$ \\ ${ }^{1}$ Department of Physics and Astronomy, University of New Mexico, Albuquerque, NM 87131, USA; tremblay@unm.edu \\ ${ }^{2}$ National Radio Astronomy Observatory, Socorro, NM 87801, USA \\ ${ }^{3}$ Astronomy Department, California Institute of Technology, Pasadena, CA 91125, USA \\ ${ }_{5}^{4}$ Naval Research Laboratory, Code 7213, Washington, DC 20375, USA \\ ${ }^{5}$ Department of Physics, Stanford University, Stanford, CA 94305, USA \\ Received 2009 December 28; accepted 2010 January 28; published 2010 February 25
}

\begin{abstract}
We present multi-frequency Very Long Baseline Array observations of J16021+3326. These observations, along with variability data obtained from the Owens Valley Radio Observatory candidate gamma-ray blazar monitoring program, clearly indicate that this source is a blazar. The peculiar characteristic of this blazar, which daunted previous classification attempts, is that we appear to be observing down a precessing jet, the mean orientation of which is aligned with us almost exactly.
\end{abstract}

Key words: galaxies: active - galaxies: evolution - galaxies: individual (J16021+3326) - galaxies: jets - galaxies: nuclei - radio continuum: galaxies

\section{INTRODUCTION}

The galaxy J16021+3326 (B1600+335) is classified as a gigahertz-peaked spectrum (GPS) radio source (Labiano et al. 2007). GPS sources are so named due to their spectral energy distribution (SED) having a radio turnover frequency of around $1 \mathrm{GHz}$. This class is principally composed of two types of sources: compact symmetric objects (CSOs) and core jets (e.g., blazars). CSOs are dual-lobed sub-kiloparsec scale sources oriented close to the plane of the sky whose emission is dominated by the hot spots where their jets ram into the surrounding medium. The common opinion is that synchrotron selfabsorption within the hot spots causes a spectral turnover of around $1 \mathrm{GHz}$ (e.g., de Vries et al. 2009), although free-free absorption has also been invoked (e.g., Bicknell et al. 2003). Core jets can be classified as GPS sources due to their highly variable SED (Tornikoski et al. 2009), or if by chance the emission is dominated by jet structures of the appropriate size to have a peak of around $1 \mathrm{GHz}$ (Scott \& Readhead 1977). Determining the reason a source is a GPS typically requires multi-frequency imaging via Very Long Baseline Interferometery (VLBI) techniques, using instruments such as the Very Long Baseline Array (VLBA), to morphologically classify the source. In principle, long-term SED variability studies could also be used to discern why a particular source is classified as a GPS (Tornikoski et al. 2009).

Previous VLBI observations of J16021+3326 by Kulkarni \& Romney (1990) and Dallacasa et al. (1995) were unable to disentangle the source structure well enough to explain why this is a GPS source. After observations at $5 \mathrm{GHz}$ in the VLBA Imaging and Polarimetry Survey (VIPS; Helmboldt et al. 2007), we decided to perform multi-wavelength follow-up with the VLBA to clarify the nature of this source.

In this paper, we have adopted a redshift for J16021+3326 of 1.1 as per Snellen et al. (2000) and Labiano et al. (2007) ${ }^{6}$. Throughout this discussion, we assume $H_{0}=73 \mathrm{~km} \mathrm{~s}^{-1} \mathrm{Mpc}^{-1}$, $\Omega_{m}=0.27, \Omega_{\Lambda}=0.73$, so 1 mas $=7.991 \mathrm{pc}$.

\footnotetext{
6 Since there are no spectroscopic data cited within these papers nor within their references, this redshift should only be taken as a possible value and spectroscopic observations should be published with a verifiable value of $z$.
}

\section{OBSERVATIONS AND DATA REDUCTION}

Multi-frequency observations of J16021+3326 were performed on 2007 February 19 with the VLBA; a summary of these observations is presented in Table 1. These observations consisted of four $8 \mathrm{MHz}$ wide intermediate frequencies (IFs) in the $C, X$, and $U$ bands with full polarization centered at 4605.5, 4675.5, 4990.5, 5091.5, 8106.0, 8176.0, 8491.0, 8590.0, $14902.5,14910.5,15356.5$, and $15364.5 \mathrm{MHz}$ at an aggregate bit rate of $256 \mathrm{Mbps}$ to maximize $(u, v)$ coverage and sensitivity. When the data in each band were combined, the three central frequencies were 4844.7, 8344.7, and 15137.5 MHz. The integrations were performed in blocks $(\sim 2$ minutes for 5 and $8 \mathrm{GHz}, \sim 7.5$ minutes for $15 \mathrm{GHz}$ ) and these blocks were spread out over a $10 \mathrm{hr}$ period to maximize $(u, v)$ coverage of the source.

Most of the calibration and initial imaging of the data were carried out by automated AIPS (Greisen 2003) and Difmap (Shepherd 1997) scripts similar to those used in reducing the VIPS $5 \mathrm{GHz}$ survey data (Helmboldt et al. 2007; Taylor et al. 2005). To summarize, flagging of bad data and calibration were performed using the VLBA data calibration pipeline (Sjouwerman et al. 2005), while imaging was performed using Difmap scripts described in Taylor et al. (2005). Final imaging was performed manually using the Difmap program, with beam sizes of $2.016 \times 2.597$ mas in position angle $-31.86,1.176 \times$ 1.557 mas in position angle -32.85 , and $0.6284 \times 0.979$ mas in position angle -29.68 for 5,8 , and $15 \mathrm{GHz}$, respectively. For polarimetry purposes, the lower two and upper two IFs in each band were paired and imaged.

$\mathrm{J} 16021+3326$ has also been observed regularly at $15 \mathrm{GHz}$ with the $40 \mathrm{~m}$ telescope at the Owens Valley Radio Observatory (OVRO) since mid-2007 as part of an ongoing candidate gamma-ray blazar monitoring program. This program monitors all 1158 sources with declination greater than $20^{\circ}$ in the CGRaBS sample (Healey et al. 2008) at least twice per week when weather conditions permit. The CGRaBS sample consists of radio sources selected as likely to be active gamma-ray emitters based on their flat radio spectra and X-ray fluxes. CGRaBS sources are predominantly known blazars, but also include a few other types. J16021+3326 is identified as a narrowline radio galaxy in the CGRaBS catalog. 
Table 1

VLBA Observations of J16021+3326

\begin{tabular}{|c|c|c|c|c|c|c|c|}
\hline $\begin{array}{l}\text { Freq. } \\
(\mathrm{GHz})\end{array}$ & Date & $\begin{array}{c}\text { Time } \\
\text { (minutes) }\end{array}$ & $\begin{array}{c}\delta v \\
(\mathrm{MHz})\end{array}$ & Pol. & IFs & $\begin{array}{c}\text { Peak } \\
\left(\mathrm{mJy} \mathrm{beam}^{-1}\right)\end{array}$ & $\begin{array}{c}\mathrm{rms} \\
\left(\mathrm{mJy} \mathrm{beam}^{-1}\right)\end{array}$ \\
\hline 4.8447 & 2007 Feb 19 & 24.9 & 32 & 4 & 4 & 685.06 & 0.15 \\
\hline 8.3447 & 2007 Feb 19 & 26.9 & 32 & 4 & 4 & 507.36 & 0.15 \\
\hline 15.138 & 2007 Feb 19 & 90.8 & 32 & 4 & 4 & 319.62 & 0.11 \\
\hline
\end{tabular}

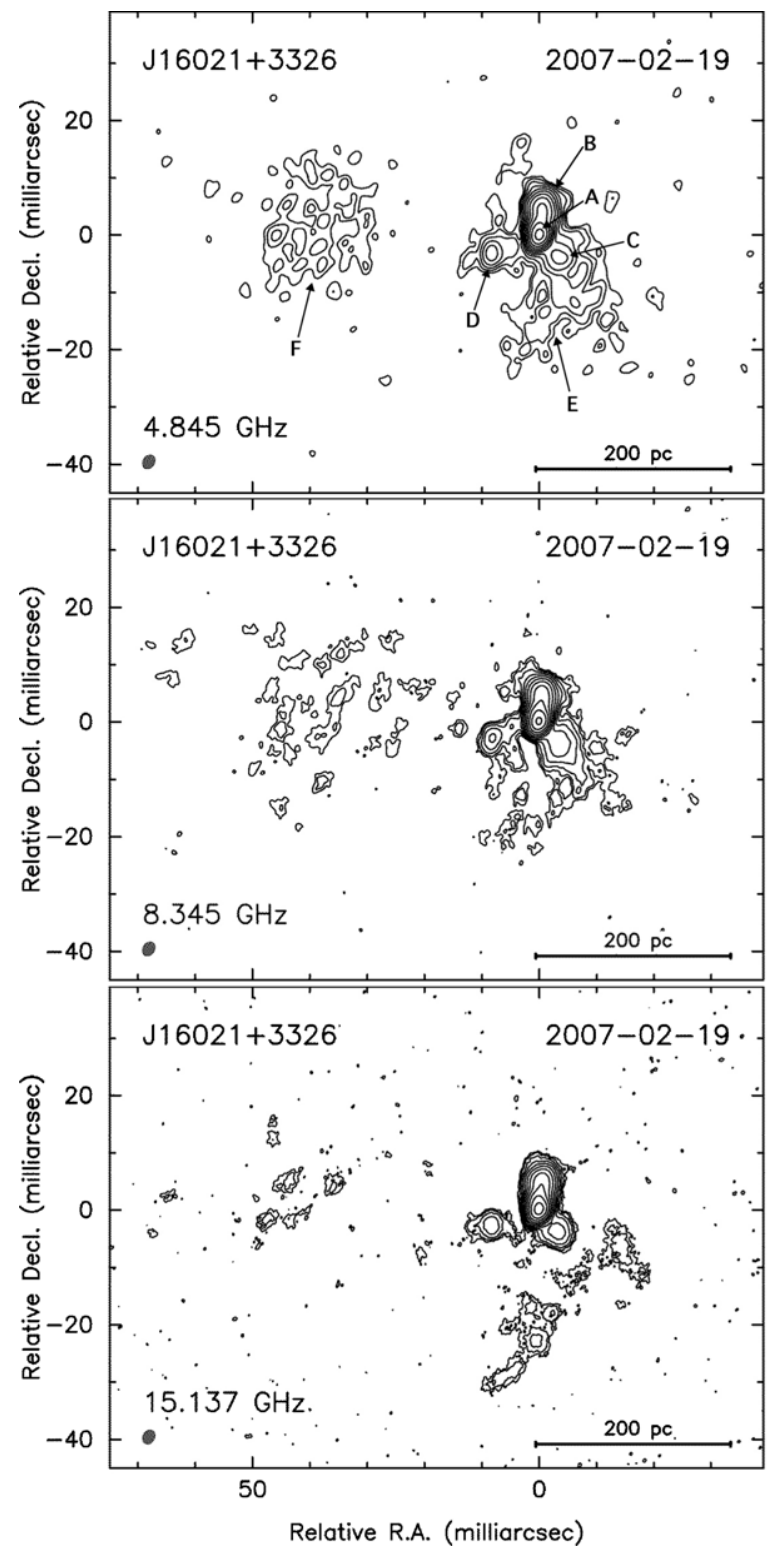

Figure 1. VLBA observations from 2007 February of J16021+3326 at frequencies of (from top to bottom) 4.845, 8.345, and $15.137 \mathrm{GHz}$. Contour levels begin at $0.5 \mathrm{mJy}, 0.5 \mathrm{mJy}$, and $0.3 \mathrm{mJy}$ respectively and increase by factors of $2^{1 / 2}$. All three of these images have been convolved with the $5 \mathrm{GHz}$ beam to enable easy comparison at matched resolution. The various components, A through $\mathrm{F}$, have been labeled on the $4.845 \mathrm{GHz}$ image, ordered such that subsequent letters correspond to decreasing peak flux density.

The OVRO $15 \mathrm{GHz}$ flux densities of J16021+3326 were measured using azimuth double switching as described in Readhead et al. (1989). The relative uncertainties in flux density result from a $5 \mathrm{mJy}$ typical thermal uncertainty in quadrature with a $1.4 \%-2 \%$ non-thermal random error contribution. The absolute flux density scale is calibrated to about $5 \%$ using the Baars et al. model for 3C 286 (Baars et al. 1977). This absolute uncertainty is not included in the plotted errors. In addition to several interruptions in the monitoring program due to hardware failures, J16021+3326 was one of the subsets of the sample affected by an unreliable pointing procedure during the period MJD 54750-54908 which resulted in spurious fluctuations in the measured flux densities. All the potentially significant fluctuations in this period were closely correlated with similar fluctuations in other unrelated sources, suggesting that the intrinsic flux density of J16021+3326 was relatively constant during this interval.

An optical $r$-band observation of J16021+3326 was also performed, using five $300 \mathrm{~s}$ exposures with the Palomar 200" telescope in 2008 July.

\section{RESULTS}

\subsection{Images}

Figures 1 and 2 show the 5, 8, and $15 \mathrm{GHz}$ VLBA images made from the 2007 February observations of J16021+3326. In Figure 1, all three images have been convolved with identical beams, both to enable simpler comparison between the maps and to highlight the detected diffuse emission at $15 \mathrm{GHz}$. The scale of Figure 2 was chosen to focus on the structure surrounding the phase center. All three images in Figure 2 are displayed at their natural resolutions to better show the complicated small-scale structure. Self-calibration was utilized when reducing these data, so all absolute positions are lost since the brightest emission was placed at the phase center. The $5 \mathrm{GHz}$ images reveal a northern elongation (B) coming from the phase center (A), and more diminished spurs toward the east (D) and southwest (E). Diffuse emission is seen $\sim 40$ mas to the east $(\mathrm{F})$, as well as surrounding the southwest spur $(\mathrm{E})$. In the $8 \mathrm{GHz}$ image, the diffuse emission is much less prominent but components A, B, C, and D are all still easily identifiable, although the southwest spur $(\mathrm{C})$ has resolved into a ring. At $15 \mathrm{GHz}$, the brightest component (A) has resolved into two distinct components (A1, A2), and the diffuse eastern emission (F) has almost completely disappeared. The eastern spur (D) is barely detected, and the southwestern spur (C) has marginally retained its ring-like structure.

The $r$-band images obtained by the Palomar 200" (Figure 3) show a faint (1.4 $\mu \mathrm{Jy}$ integrated over a 3."6 aperture, for an absolute magnitude of 21.0) galaxy with evidence of a disturbed morphology, which is consistent with previous optical observations by Stickel \& Kuehr (1996).

\subsection{Spectral Index Distribution}

The 8 and $15 \mathrm{GHz}$ images were matched in resolution in order to obtain a spectral index distribution across the source, where we take $F_{v} \propto v^{\alpha}$, which was overlaid onto $5 \mathrm{GHz}$ contours to highlight the placement of the distribution within the source structure (Figure 4). Averaged spectral indices for components A1, A2, B, C, D, and F and can be found in Table 2. In summary, 

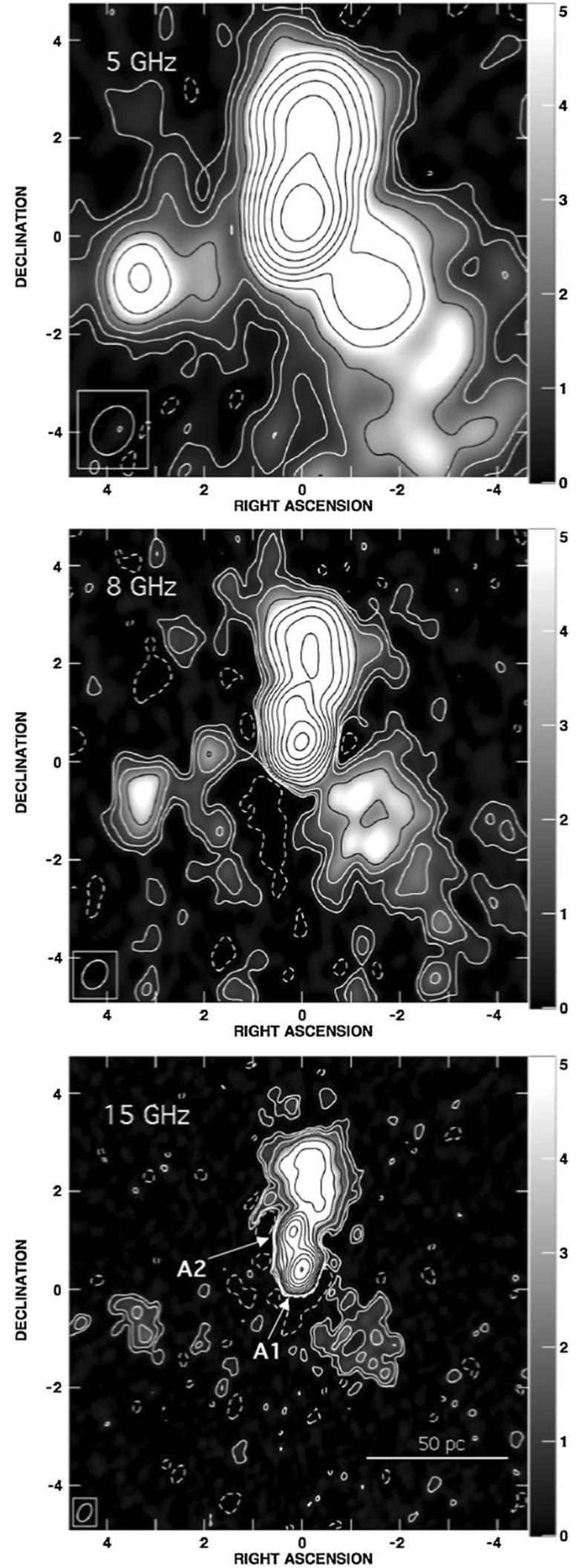

Figure 2. Zoomed in view of the VLBA observations from 2007 February of $\mathrm{J} 16021+3326$ at frequencies of (from top to bottom) 4.845, 8.345, and $15.137 \mathrm{GHz}$. Contour levels begin at $0.35 \mathrm{mJy}, 0.4 \mathrm{mJy}$, and $0.3 \mathrm{mJy}$ respectively and increase by factors of $2^{1 / 2}$. The gray scale shows the details of the emission between $0 \mathrm{mJy}$ and $5 \mathrm{mJy}$.

the only component exhibiting a flat spectrum (i.e., an $\alpha \approx 0$ ) is $\mathrm{A} 2$.

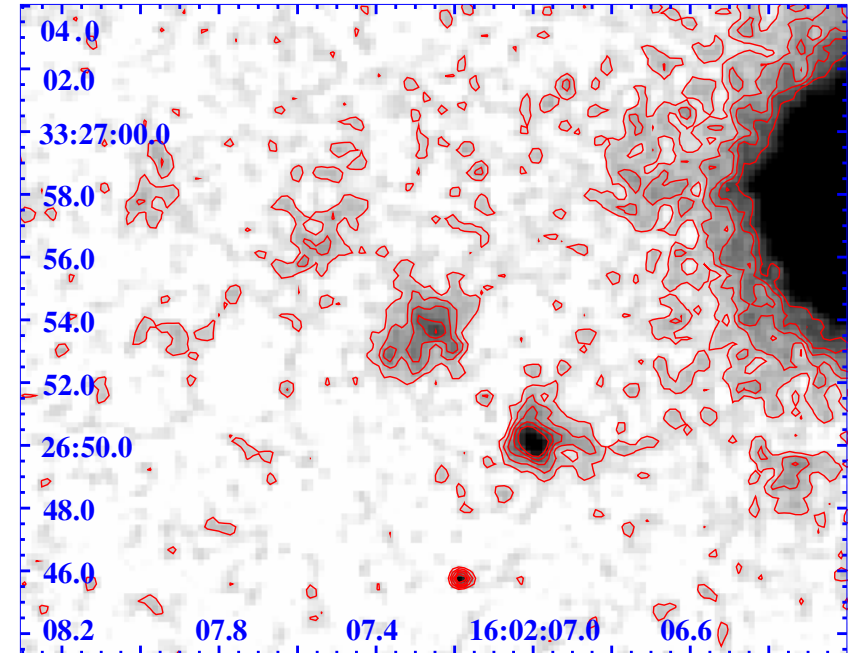

Figure 3. Optical $r$-band image of $\mathrm{J} 16021+3326$ (center of field). This image was taken with the Palomar $200^{\prime \prime}$ telescope over five $300 \mathrm{~s}$ exposures in 2008 July. The central 1.1 has a flux density of $0.73 \mu \mathrm{Jy}$, while the entire galaxy, integrated over a $3: 6$ aperture, has a flux density of $1.4 \mu \mathrm{Jy}$.

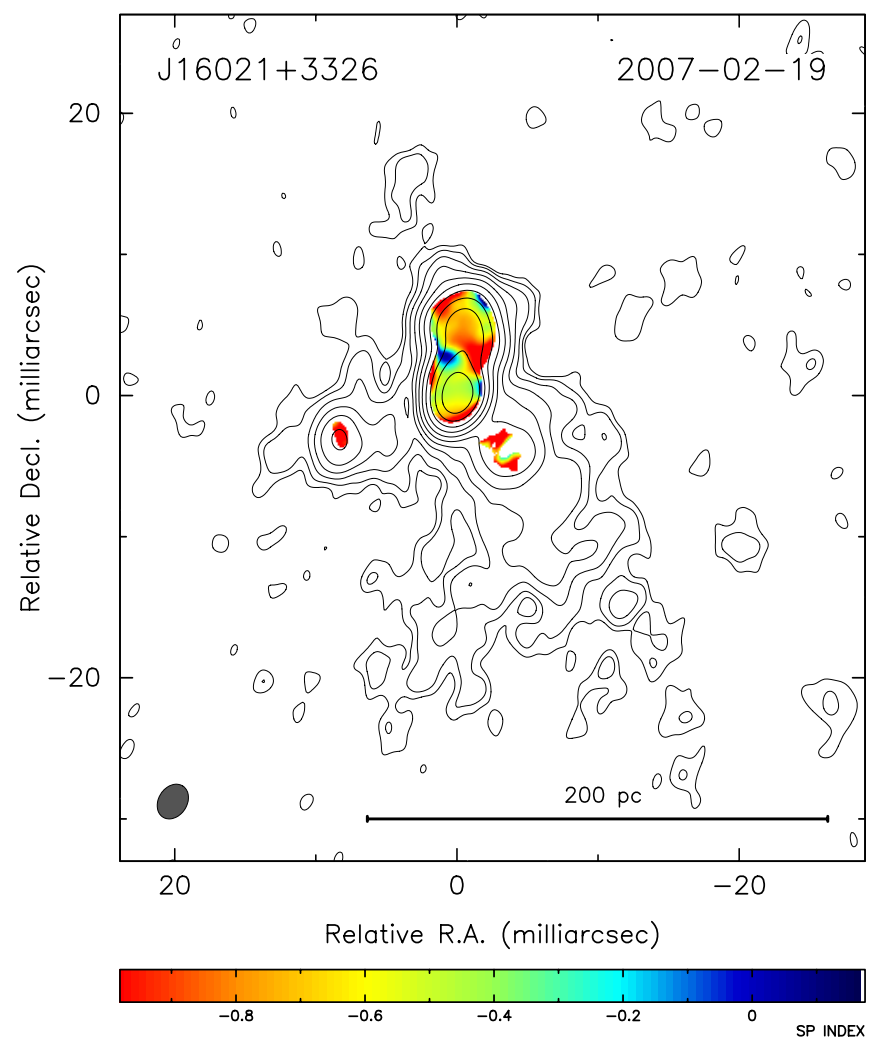

Figure 4. Multi-frequency observations of J16021+3326; $5 \mathrm{GHz}$ contours overlaid on an $8-15 \mathrm{GHz}$ spectral index image. The flat-spectrum $(\alpha \approx-0.05)$ compact component likely identifies the core of the source.

\subsection{Polarization}

Polarization was detected at both 8 and $15 \mathrm{GHz}$ with up to $14.9 \% \pm 2.0 \%$ and $5.7 \% \pm 0.7 \%$ polarization, respectively. Figure 5, showing the polarized flux from the IF pair centered at $15.3605 \mathrm{GHz}$, provides an example of the polarization maps obtained. Components A, B, C, and D all exhibit polarization in the four IF pairs, although the pair centered at $14.9065 \mathrm{GHz}$ detected significantly less polarization, the reason for which is unknown. The three IF pairs that detected stronger polarization were used to compute rotation measures (RMs) across the source 


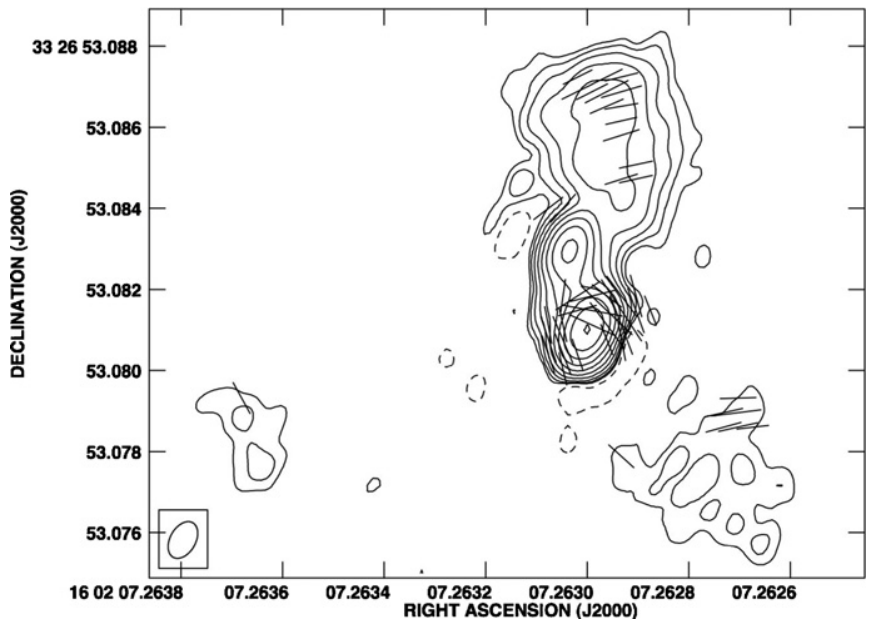

Figure 5. Uncorrected polarization electric field $(E)$ vectors from one of the two averaged IF pairs at $15 \mathrm{GHz}$ overlaid onto $15 \mathrm{GHz}$ total intensity contours. The vector lengths are proportional to the polarized flux density and 1 mas $=$ $7.58 \times 10^{-4} \mathrm{Jy} \mathrm{beam}^{-1}$. Contour levels begin at $0.6 \mathrm{mJy}$ and increase by factors of $2^{1 / 2}$. This is an example of how polarization was detected throughout the source, although the exact positions were frequency dependent.

Table 2

Eight to $15 \mathrm{GHz}$ Spectral Index of Various Components of J16021+3326

\begin{tabular}{lcc}
\hline \hline Component & Spectral Index $(\alpha)^{\mathrm{a}}$ & $\mathrm{rms}^{\mathrm{b}}$ \\
\hline A1 & -0.54 & 0.08 \\
A2 & -0.05 & 0.07 \\
B & -0.75 & 0.07 \\
C & -1.05 & 0.11 \\
D & -0.97 & 0.35 \\
F & -0.79 & 0.72 \\
\hline
\end{tabular}

\section{Notes.}

${ }^{\text {a }}$ Here we use the convention of $F_{v} \propto v^{\alpha}$, and $\alpha$ is averaged over the component using the $8 \mathrm{GHz}$ and $15 \mathrm{GHz}$ data.

$\mathrm{b}$ This is the rms of $\alpha$ for the averaged area.

(Figure 6) wherever polarization was detected at all three pairs by fitting the change in polarization angle $(\beta)$ to $\mathrm{RM}=\frac{\beta}{\lambda^{2}}$, with values ranging from -984 to $1426 \mathrm{rad} \mathrm{m}^{-2}$. The $\mathrm{RM}$ is dependent on the magnetic field strength $(B)$ and the electron number density $\left(n_{e}\right)$ by

$$
\mathrm{RM}=\frac{e^{3}}{2 \pi m_{e}^{2} c^{4}} \int_{0}^{s} n_{e} B d s,
$$

where $e$ and $m_{e}$ are the charge and mass of the electron, respectively, and $c$ is the speed of light. These RMs were then used to calculate the magnetic field polarization angle corrected for Faraday rotation (the inset in Figure 6). It is worth noting that the RM changes sign across A1, which is indicative of a reversal of the magnetic field as seen in the above equation.

\subsection{Variability}

The OVRO $15 \mathrm{GHz}$ light curve for J16021+3326 is shown in Figure 7, along with scaled and offset light curves for the bright, constant-flux sources 3C 48, 3C 286, and DR 21 for reference. The median flux density of J16021+3326 is $858 \mathrm{mJy}$ and the root mean square (rms) is $36 \mathrm{mJy}$, or $4.2 \%$ of the median. The rms fluctuations of $3 \mathrm{C} 48,3 \mathrm{C} 286$, and DR 21 are $1.7 \%, 1.3 \%$, and $1.2 \%$ of the median flux density, respectively. The peak-topeak variation is $133 \mathrm{mJy}$, or $15.5 \%$ of the median. We conclude that the variation in flux density of $\mathrm{J} 16021+3326$ results from

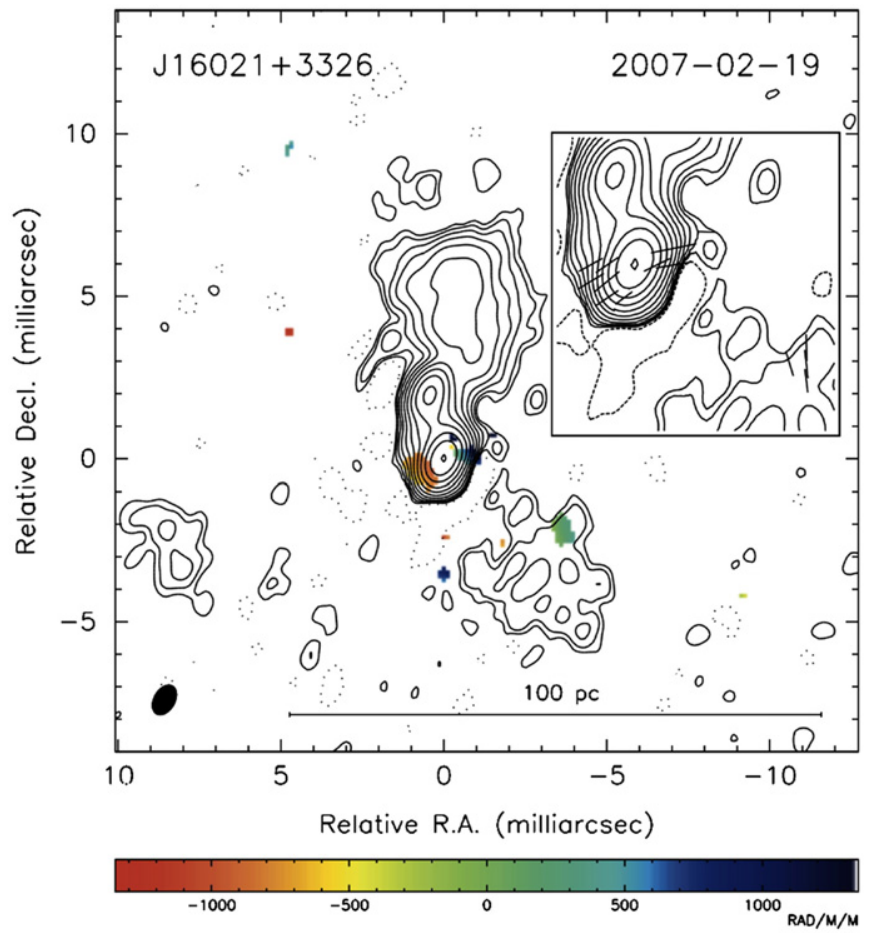

Figure 6. RM map computed from polarization angles measured at three frequencies overlaid onto $15 \mathrm{GHz}$ contours. Contour levels begin at $0.3 \mathrm{mJy}$ and increase by factors of $2^{1 / 2}$. The inset shows the corrected polarization angle of the magnetic fields $(B)$ of the source.

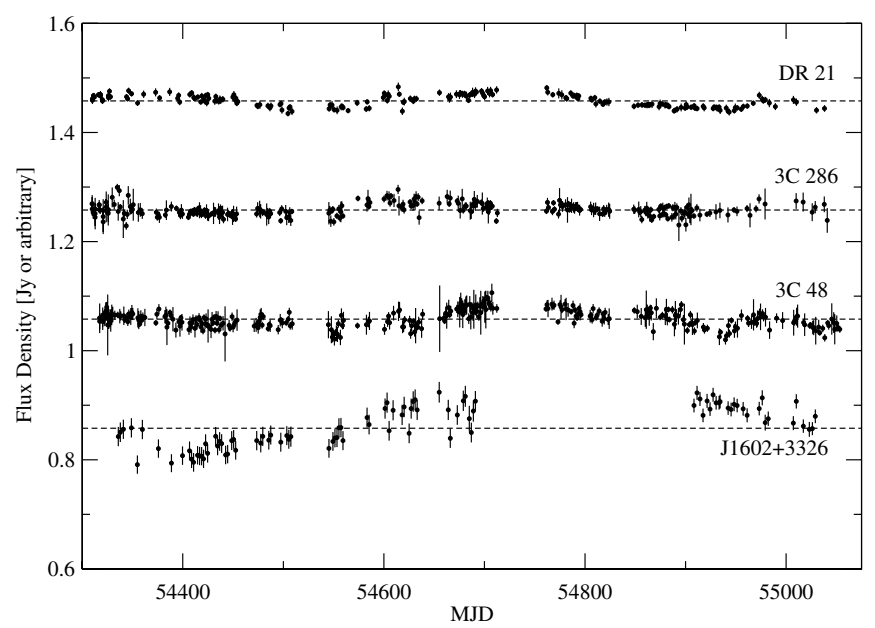

Figure 7. OVRO $40 \mathrm{~m} 15 \mathrm{GHz}$ light curve for J16021+3326 (bottom, in Jy). Shown for reference are scaled and arbitrarily offset light curves for three stableflux density sources DR 21, 3C 286, and 3C 48. The reference curves were scaled to the same median flux density as J16021+3326, then offset for display. Dashed lines indicate the median of each light curve. Slow variation seen in the flux density of $\mathrm{J} 16021+3326(15.5 \%$ peak to peak, $4.2 \% \mathrm{rms})$ is greater in magnitude than the systematic fluctuations observed in the stable sources (6\%-9\% peak to peak, $1.2 \%-1.7 \% \mathrm{rms})$.

actual source variations rather than systematic instrumental fluctuations. These observed fluctuations are consistent with the variability of the flux density (up to $60 \%$ peak to peak at $5 \mathrm{GHz}$ over a 6 year period) noted by Dallacasa et al. (1995).

\section{DISCUSSION}

\subsection{Core Identification}

The unresolved, flat-spectrum $(\alpha \sim-0.05)$, unpolarized component seen in Figure 4 corresponds with A2 (Figure 2) 
and is consistent with the emission from the base of the radio jet, commonly referred to as the core (Begelman et al. 1984). The remainder of the emission surrounding the core (A1, B, C, D, E, and F) all exhibit steeper spectra $(\alpha \sim-0.54$ to -1.05$)$ and are consistent with the identification of these components as jets or hot spots.

\subsection{Morphology}

Taken alone, the components A1, A2, and B look very much like a CSO, in which the core feeds into two symmetric lobes to the north and south. However, this scenario does not readily explain the remaining components, with the positions of $\mathrm{D}$ and $\mathrm{F}$ being particularly difficult to reconcile. This source also does not resemble a simple core jet since there are extended components all around the core. Clearly, imaging alone is not enough to determine the proper classification of this complicated source.

The OVRO variability data argue against J16021+3326 being a CSO. Since the jets associated with CSOs are oriented close to the plane of the sky, there is little to no relativistic beaming of their emission expected and the emission is dominated by the extended hot spots where the jets terminate. The flux densities of five CSOs were observed to fluctuate over an 8 month period with an average rms of $0.7 \%$ (Fassnacht \& Taylor 2001), while the two core-jet sources observed over the same period had an average rms of $3 \%$. Therefore, the observed variability of this source, $15.5 \%$ peak to peak and $4.2 \%$ rms over a 2 year period, strongly supports the blazar scenario.

Lastly, the polarization data also argue against J16021+3326 being a CSO. Since the jets of CSOs are oriented close to the plane of the sky and are on the size scale of $10 \mathrm{~s}$ of parsecs, they should be viewed through the torus according to active galactic nucleus unification models (Antonucci 1993). This torus can act as a Faraday screen, thus depolarizing the synchrotron radiation as it leaves the galaxy (Peck \& Taylor 2000; Gugliucci et al. 2007). Very few CSOs have been observed to be polarized, and those that only exhibit polarization in the oncoming jet. Since we observe polarized flux all around the core of this source, it is unlikely to be a CSO. The derived RMs also lie within the typical blazar range of 500 to several thousand $\mathrm{rad} \mathrm{m}^{-2}$ (Zavala \& Taylor 2004).

While there seems to be plenty of evidence of the blazar nature of $\mathrm{J} 16021+3326$, it is far from a conventional member of the class. Typically, one would expect to see a one-sided jet coming out of the core, while in this case there appears to be jet emission all around the core. Precession, observed in other radio sources (e.g., SS 433; Dubner et al. 1998), provides a simple explanation for this unconventional morphology from a blazar. If the jet is directed exceptionally close to the line of sight, any precession would make the jet appear to spiral around the core instead of producing the more familiar core-jet morphology typically exhibited by blazars. The structure observed in J16021+3326 (Figure 1) is consistent with knots moving along a spiraling jet and/or foreground hot spot projection.

J16021+3326 does display two non-blazar-like characteristics since it both appears to lack broad-line emission and it is optically extended (i.e., not a core-dominated optical quasar). The first of these appears to be a non-issue since the spectral classification is not well supported in the literature and yields a second reason that a spectrum of this source should be observed and published in the future. As far as the optical classification is concerned, there are other examples of sources optically classified as galaxies exhibiting blazar-like behavior in the radio (e.g., 3C 111 which exhibits superluminal motion and high variability; Cohen et al. 2007). It is possible that this source is obscured by dust in the optical, but unaffected at radio wavelengths.

\section{CONCLUSIONS}

Analysis of multi-frequency $(5,8$, and $15 \mathrm{GHz})$ VLBA data shows a compact, flat-spectrum $(\alpha \sim-0.05)$ component that we identify as the core of J16021+3326 surrounded by steeperspectrum $(\alpha \sim-0.5$ to -1.0$)$ polarized regions of emission. Also, data from the OVRO candidate gamma-ray blazar monitoring program reveal a relatively high level of variability ( $15.5 \%$ peak to peak and $4.2 \%$ rms over a 2 year period). This evidence, coupled with the detection of polarization in most components other than the core, indicates that J16021+3326 is a blazar. The peculiar structure observed is possibly due to the jet, presumably pointed close to the line of sight, precessing around and tracing out a spiral on the sky.

The National Radio Astronomy Observatory is a facility of the National Science Foundation operated under cooperative agreement by Associated Universities, Inc.

Facilities: VLBA

\section{REFERENCES}

Antonucci, R. 1993, ARA\&A, 31, 473

Baars, J. W. M., Genzel, R., Pauliny-Toth, I. I. K., \& Witzel, A. 1977, A\&A, 61,99

Begelman, M. C., Blandford, R. D., \& Rees, M. J. 1984, Rev. Mod. Phys., 56, 255

Bicknell, G. V., Saxton, C. J., \& Sutherland, R. S. 2003, Publ. Astron. Soc. Aust., 20, 102

Cohen, M. H., Lister, M. L., Homan, D. C., Kadler, M., Kellermann, K. I., Kovalev, Y. Y., \& Vermeulen, R. C. 2007, ApJ, 658, 232

Dallacasa, D., Fanti, C., Fanti, R., Schilizzi, R. T., \& Spencer, R. E. 1995, A\&A, 295, 27

de Vries, N., Snellen, I. A. G., Schilizzi, R. T., Mack, K.-H., \& Kaiser, C. R. 2009, A\&A, 498, 641

Dubner, G. M., Holdaway, M., Goss, W. M., \& Mirabel, I. F. 1998, AJ, 116, 1842

Fassnacht, C. D., \& Taylor, G. B. 2001, AJ, 122, 1661

Greisen, E. W. 2003, Astrophys. Space Sci. Libr., 285, 109

Gugliucci, N. E., Taylor, G. B., Peck, A. B., \& Giroletti, M. 2007, ApJ, 661, 78

Healey, S. E., et al. 2008, ApJS, 175, 97

Helmboldt, J. F., et al. 2007, ApJ, 658, 203

Kulkarni, V. K., \& Romney, J. D. 1990, in Compact Steep-Spectrum \& GHZpeaked Spectrum Radio Sources, ed. C. Fanti, R. Fanti, \& C. P. O’Dea (Bologna: Consiglio Nazionale delle Richerche, Istituto di Radioastronomia), 85

Labiano, A., Barthel, P. D., O’Dea, C. P., de Vries, W. H., Pérez, I., \& Baum, S. A. 2007, A\&A, 463, 97

Peck, A. B., \& Taylor, G. B. 2000, ApJ, 534, 90

Readhead, A. C. S., Lawrence, C. R., Myers, S. T., Sargent, W. L. W., Hardebeck, H. E., \& Moffet, A. T. 1989, ApJ, 346, 566

Scott, M. A., \& Readhead, A. C. S. 1977, MNRAS, 180, 539

Shepherd, M. C. 1997, in ASP Conf. Ser. 125, Astronomical Data Analysis Software and Systems VI, ed. G. Hunt \& H. E. Payne (San Francisco, CA: ASP), 77

Sjouwerman, L. O., Mioduszewski, A. J., \& Greisen, E. W. 2005, in ASP Conf. Ser. 340, Future Directions in High Resolution Astronomy, ed. J. Romney \& M. Reid (San Francisco, CA: ASP), 613

Snellen, I. A. G., Schilizzi, R. T., Miley, G. K., de Bruyn, A. G., Bremer, M. N., \& Röttgering, H. J. A. 2000, MNRAS, 319, 445

Stickel, M., \& Kuehr, H. 1996, A\&AS, 115, 11

Taylor, G. B., et al. 2005, ApJS, 159, 27

Tornikoski, M., et al. 2009, Astron. Nachr., 330, 128

Zavala, R. T., \& Taylor, G. B. 2004, ApJ, 612, 749 